\title{
BMC Dermatology Reviewer Acknowledgement, 2015
}

\author{
Guangde Tu
}

\section{Contributing reviewers}

The editors of BMC Dermatology would like to thank all our reviewers who have contributed their time to the journal in Volume 15 (2015).

\author{
Rania Abdel Hay \\ Egypt \\ Ilknur Altunay \\ Turkey \\ Joao Guilherme Alves \\ Brazil \\ Christian Apfelbacher \\ Germany
}

Robert Bransfield

USA

Luis Cabero

Spain

Barbara Castelnuovo

Uganda

Mutlu Cayirli

Turkey

Sheau-Chiou Chao

Taiwan

Mingyi Chen

USA

Chien-Ping Chiang

Taiwan

Kyung-Soo Chun

Republic of Korea

Giovanni Di Zenzo

Italy

\author{
Seval Dogruk Kacar \\ Turkey \\ Jacquelyn Dosal \\ USA \\ Félix Fanjul-Vélez \\ Spain \\ Steven Feldman \\ USA
}

Mari-Ann Flyvholm

Denmark

Claire Fuller

United Kingdom

Pratik Gahalaut

India

Xiaolian Gu

Sweden

Carolyn Heckman

USA

Elke Heiss

Austria

Kedar Inamdar

USA

Liselotte Jensen

USA

Peter Kaskel

Germany

\section{Leon Kircik \\ USA}

\section{Lissy Krishnan \\ India}

\section{Bhushan Kunar}

India

Francesco Lacarrubba
Italy

Min-Geol Lee

Republic of Korea

Jason Lee

Philippines

Hauryueh Lee

Singapore

Jacob Levitt

USA

\section{Marie Loden}

Sweden

Lois Loescher

USA

Gurusamy R. Manoharan

India

Amy McMichael

USA

Luigi Naldi

Italy

Correspondence: guangde.tu@biomedcentral.com

Room 1504-1505 Financial Plaza, No. 333 Jiujiang Road, Huangpu District,

Shanghai 200001, China 
Alex Ortega-Loayza

USA

Jack S. Resneck

USA

Aristóteles Rosmaninho

Portugal

Gerhard Schmid-Ott

Germany

Marcus Schmitt-Egenolf

Sweden
Ali Mohammad Sharifi

Iran

Johanna Stoevesandt

Germany

Michael Sweeney

USA

Yayoi Tada

Japan

Wayne Tam

USA
Martin Tichy

Czech Republic

Jane Tomimori

Brazil

Marit B. Veierød

Norway

Hywel Williams

United Kingdom

Nabiha Yusuf

USA 